\title{
Identification of up-regulated and down-regulated cis-natural antisense transcripts in the human B lymphoblastic cell line IM-9 after X-ray irradiation
}

\author{
MITSURU CHIBA $^{1}$, TOMISATO MIURA ${ }^{2}$, KOSUKE KASAI $^{2}$, SATORU MONZEN $^{3}$, \\ IKUO KASHIWAKURA ${ }^{3}$, HIROSHI YASUE $^{4}$ and TOSHIYA NAKAMURA ${ }^{1}$ \\ Departments of ${ }^{1}$ Biomedical Sciences, ${ }^{2}$ Pathologic Analysis, and ${ }^{3}$ Radiological Life Sciences, \\ Division of Medical Life Sciences, Hirosaki University Graduate School of Health Sciences, Hirosaki, Aomori 036-8564; \\ ${ }^{4}$ Animal Genome Research Unit, National Institute of Agrobiological Sciences, Tsukuba, Ibaraki 305-0901, Japan
}

Received October 20, 2011; Accepted February 7, 2012

DOI: $10.3892 / \mathrm{mmr} .2012 .787$

\begin{abstract}
Ionizing radiation (IR) causes DNA injury and induces multiple signal mechanisms, including the regulation of DNA repair, the cell cycle and gene expression through the activation of p53-related pathways. Cis-natural antisense transcripts (cis-NATs), which are transcribed from the DNA strand opposite to that for mRNA of the gene, are recognized as important regulators of gene expression in eukaryotic cells, but the effects on cis-NAT expression by IR are unknown to date. Therefore, we investigated the effects of $\mathrm{X}$-ray irradiation on cis-NAT expression together with mRNA expression using a human B lymphoblast cell line (IM-9), a custom-microarray and strand-specific RT-qPCR. Eighteen, 33 and 106 mRNAs were demonstrated to be differentially expressed in IM-9 cells after 1,2 and 4 Gy irradiation, respectively, as compared to 0 Gy by microarray analysis (fold change, FC $>2.0$ ). On the other hand, 10, 22 and 43 NATs were demonstrated to be differentially expressed in IM-9 cells after 1, 2 and 4 Gy irradiation, respectively, as compared to 0 Gy by microarray analysis (FC > 2.0). Among these mRNAs/NATs, the IR dosedependent up-regulation of mRNAs and cis-NATs of MDM2 and CDKN1A were confirmed by strand-specific RT-qPCR. Additionally, the cis-NATs of MDM2 were indicated to be localized in the cytoplasm, while cis-NATs of CDKN1A were located in the nucleus and cytoplasm. In conclusion, the radiation-responsive cis-NATs in conjunction with mRNAs were identified for the first time in the present study. It is possible that these cis-NATs regulate the gene expression in
\end{abstract}

Correspondence to: Dr Mitsuru Chiba, Department of Biomedical Sciences, Division of Medical Life Sciences, Hirosaki University Graduate School of Health Sciences, 66-1 Hon-cho, Hirosaki, Aomori 036-8564, Japan

E-mail: mchiba32@cc.hirosaki-u.ac.jp

Key words: ionizing radiation, natural antisense transcripts, B lymphoblast cell, MDM2, CDKN1A a post-transcriptional fashion. The IR dose-dependent up- and down-regulation of these mRNAs/cis-NATs may be a marker for ionizing radiation.

\section{Introduction}

Ionizing radiation (IR) is effectively used for the diagnosis and treatment of various types of cancer in the medical field. However, recently, radiation exposure accidents in radiation therapy and at nuclear power plants have occurred. Therefore, it is important to study the effect of IR on living organisms to minimize the influence of accidental exposures.

IR exposure is shown to cause two distinct phenomena: one is DNA injury and the other is non-DNA injury, such as cell membrane disorder and lipid peroxidation. As a DNA injury response, poly (ADP-ribose) polymerase (PARP) and DNA-dependent protein kinase repair complex (DNA-PK) are activated by double-strand breaks of DNA to engage in DNA repair $(1,2)$. In addition, ATM, which is known as the causative gene, when affected, of Ataxia telangiectasia, is activated in response to DNA injury in order to initiate phosphorylation of p53. p53 was found to up-regulate mRNA expression of GADD45, MDM2, CDKN1A ( $\left.p 21^{C i p l}\right)$ and BAX to control the cell cycle, DNA repair and apoptosis (3-5). As a non-DNA injury phenomena, cell membrane disorder and lipid peroxidation have been observed, which induce activation of signal transduction pathways, such as those with protein kinase $\mathrm{C}$ (PKC), c-Jun $\mathrm{NH}_{2}$-terminal kinase (JNK), ceramide and/or mitogen-activated protein kinase (MAPK) (3). Recent studies have revealed that IR generates reactive oxygen species (ROS) and reactive nitrogen species (RNS) in cells, which leads to DNA injury at random sites as well as the non-DNA injury phenomena $(3,6)$.

Cis-natural antisense transcripts (cis-NATs), which are transcribed from the DNA strand opposite to the sense strand, have been discovered by full-length cDNA sequencing analysis in the human and mouse $(7,8)$. In a recent study, cis-NATs were demonstrated to be involved in the control of gene expression in a eukaryote (9). For example, it has been shown that the cisNATs of the BACE1 gene are involved in the stability of the 
Table I. Primer sequences for strand-specific RT-qPCR.

\begin{tabular}{llcc}
\hline Primer name & \multicolumn{1}{c}{ Sequence } & Size (mer) & PCR products (bp) \\
\hline$M D M 2$-forward & 5'-AGACAACCAATTCAAATGATTGTGC-3' & 25 & 60 \\
$M D M 2$-reverse & 5'-CTCTTATAGACAGGTCAACTAGG-3' & 23 & 60 \\
$C D K N 1 A$-forward & 5'-TTGATTAGCAGCGGAACAAGG-3' & 21 & 97 \\
CDKN1A-reverse & 5'-TCCATAGCCTCTACTGCCA-3' & 19 & 18 \\
ACTB-forward & 5'-CCAACCGCGAGAAGATGA-3' & 20 & 120 \\
ACTB-reverse & 5'-CCAGAGGCGTACAGGGATAG-3' & 18 & \\
$E G F P$-forward & 5'-CAGCAGAACACCCCCATC-3' & 20 & \\
$E G F P$-reverse & 5'-GAACTCCAGCAGGACCATGT-3' & & \\
\hline
\end{tabular}

BACE1 mRNA by forming RNA duplex with BACE1 mRNA (10), and that the cis-NATs of the BCMA gene are involved in the control of gene expression through translational inhibition of $B C M A$ mRNA (11). Furthermore, it has been shown that the cis-NATs of the $C D K N 2 B$ gene are involved in the control of epigenetic gene expression of the $C D K N 2 B$ gene through heterochromatin formation in leukemia cells (12). In addition, differential cis-NAT expression patterns were observed between cancerous tissues and their corresponding non-cancerous tissues obtained from colorectal and breast cancers $(13,14)$. However, the effects on cis-NAT expression by IR are unknown to date.

In the present study, the cis-NATs, up- and down-regulated by X-ray irradiation, were investigated using the human B lymphoblastic cell line IM-9 having a high sensitivity to IR, and using a custom-microarray containing human sense/ antisense probes for approximately 21,000 genes.

\section{Materials and methods}

Cell line and cell culture. The human B lymphoblastic cell line IM-9 (JCRB0024) was purchased from the Health Science Research Resources Bank (Osaka, Japan). The IM-9 cells were cultured in RPMI-1640 medium, supplemented with 10\% fetal bovine serum (FBS), $100 \mathrm{U} / \mathrm{ml}$ penicillin and $100 \mu \mathrm{g} /$ $\mathrm{ml}$ streptomycin, at $37^{\circ} \mathrm{C}$ in an atmosphere of $5 \% \mathrm{CO}_{2}$. The IM-9 cells were seeded onto 6-well plates at a concentration of $2 \times 10^{5}$ cells/well for X-ray irradiation experiments.

$X$-ray irradiation. The IM-9 cells plated on the wells were cultured as described above for $24 \mathrm{~h}$ prior to X-ray irradiation. Irradiation $(150 \mathrm{kVp}, 20 \mathrm{~mA}, 0.5-\mathrm{mm}$ aluminium and $0.3-\mathrm{mm}$ copper filters) was performed using an X-ray generator (MBR-1520R-3; Hitachi Medical Corporation, Tokyo, Japan) at a distance of $45 \mathrm{~cm}$ between the focus and the target. The dose rate was $80 \mathrm{cGy} / \mathrm{min}$. The dose was monitored with a thimble ionization chamber that was placed next to the sample during the irradiation. The irradiated cells were further cultured for $24 \mathrm{~h}$ under the same conditions, and then collected by centrifugation. The cells thus collected were frozen in liquid nitrogen and stocked at $-80^{\circ} \mathrm{C}$ until being used for RNA extraction.

RNA extraction. For microarray analysis, total RNAs were extracted from the cells using ISOGEN II (Nippon Gene,
Tokyo, Japan) according to the manufacturer's instructions. The qualities and concentrations of the RNAs extracted were examined using the NanoDrop Spectrophotometer (Thermo Scientific, Wilmington, DE, USA) according to the manufacturer's instructions. In addition, the integrities of the RNAs were examined using Agilent Bioanalyzer (Agilent Technologies, CA, USA), according to the manufacturer's instructions. The isolation of nuclear RNA and cytoplasmic RNA separately from the cells was performed using PARIS kit (Ambion, Austin, TX, USA) according to the manufacturer's instructions.

Microarray analysis. Cyanine 3 (Cy3)-labeled cDNA was synthesized from $10 \mu \mathrm{g}$ total RNA of irradiated or non-irradiated samples using a LabelStar Array kit (Qiagen, Valencia, CA, USA), Cy3-dUTP (GE Healthcare, Buckinghamshire, UK) and random nonamer primer. The sense and antisense sequences of $60 \mathrm{nt}$ custom-designed were arranged in an Agilent 44K x 4 system [20882 ORFs: Agilent eArray Design ID $=19052$ produced by Tsukuba GeneTech Laboratory (Ibaraki, Japan)] (13). Agilent 44K x 4 human sense/antisense custom-microarray slides were hybridized with the Cy3-labeled cDNA ( $2 \mu \mathrm{g})$ in a hybridization solution prepared with a GE Hybridization kit (Agilent Technologies). The $\mathrm{Cy} 3$ fluorescence signal images on the slides were obtained by a DNA microarray scanner (Agilent Technologies) and processed using the Feature Extraction version 8.1 software based on the instructions from Agilent Technologies.

The expression data thus obtained were processed using GeneSpring GX ver. 10 software (Agilent Technologies) in order to carry out a normalization to the 75 percentile of all values on the respective microarrays, followed by normalization of the median expression levels of all samples. The expression profiles of the mRNAs and cis-NATs were compared, based on the fold-change of the values $(>100)$ of the respective genes. The up- and down-regulated transcripts of $>2$-fold at 1,2 and 4 Gy IR against 0 Gy were selected.

Strand-specific RT- $q P C R$. Total RNAs from irradiated and nonirradiated IM-9 cells were used for strand-specific RT-qPCR of mRNAs and cis-NATs. An aliquot of each RNA sample was mixed with an amount of RNA fragment (218 nucleotides) synthesized from the pEGFP-C1 vector (Invitrogen, CA, USA) to attain a final amount of $5 \times 10^{-5} \mathrm{pmol} / 10 \mu \mathrm{g}$ total RNA. These 
RNAs were subjected to synthesis of the first-strand cDNA only from mRNA or cis-NATs using reverse or forward primer (Table I), and AMV Reverse Transcriptase (Promega, Madison, WI, USA) together with $A C T B$ reverse primer as the internal control and $E G F P$ forward primer as the external control, according to the procedure recommended by Promega (15). The mixtures were then incubated at $50^{\circ} \mathrm{C}$ for $60 \mathrm{~min}$. The resulting cDNAs were incubated at $99^{\circ} \mathrm{C}$ for $5 \mathrm{~min}$ and at $37^{\circ} \mathrm{C}$ for $60 \mathrm{~min}$ with RNase A to digest RNA. The first-strand cDNAs were first subjected to ordinary PCR using the primer sets described in Table I in order to confirm whether the fragments amplified in the PCR were the ones derived from the target transcripts based on fragment size in $4 \%$ agarose gel electrophoresis.

The first-strand cDNAs derived from mRNAs or $c i s$-NATs were used as a template for quantitative PCR (qPCR) using Power SYBR Green PCR Master Mix (Applied Biosystems, CA, USA) and primer pair sets described in Table I. The qPCRs were performed using an Applied Biosystems StepOne Plus Real-Time PCR system (Applied Biosystems) under the conditions of $10 \mathrm{~min}$ at $95^{\circ} \mathrm{C}$ followed by 40 cycles each of $95^{\circ} \mathrm{C}$ for $15 \mathrm{sec}$ and $60^{\circ} \mathrm{C}$ for $60 \mathrm{sec}$, following the procedure recommended by the manufacturer. In order to compare the PCR results, the values for mRNAs and cis-NATs were normalized based on the values of the control.

Strand-specific RT-qPCR data thus obtained were statistically examined using one-way analysis of variance (one-way ANOVA). Within each data set, post hoc multiple comparisons were run with Tukey's Honestly Significant Difference test to determine the statistical significance for differences between each pair of means. Differences were considered significant at a value of $\mathrm{p}<0.05$.

Strand-specific RT-PCR for the intracellular localization of transcripts. To know the intracellular localization of mRNAs and cis-NATs, RT-PCR was performed using strand-specific cDNAs synthesized from total RNA (250 ng), cytoplasmic RNA (200 ng) and nuclear RNA (50 ng). These cDNAs were subjected to PCR in a $45 \mu 1$ reaction mixture containing $1 \mathrm{x}$ buffer, $1.5 \mathrm{mM} \mathrm{MgCl} 2,0.1 \mathrm{mM}$ of each dNTP, $0.025 \mathrm{U} / \mu \mathrm{l}$ of Biotaq HS DNA polymerase (Nippon Genetics, Tokyo, Japan), $0.5 \mu \mathrm{M}$ of the primer pairs (Table I) and the cDNA template. The PCRs were performed using the Veriti 96-Well Thermal Cycler (Applied Biosystems) under the conditions of $7 \mathrm{~min}$ at $95^{\circ} \mathrm{C}$, followed by 35 cycles each of $95^{\circ} \mathrm{C}$ for $30 \mathrm{sec}, 60^{\circ} \mathrm{C}$ for $30 \mathrm{sec}$ and $72^{\circ} \mathrm{C}$ for $30 \mathrm{sec}$ and a final elongation step at $72^{\circ} \mathrm{C}$ for $5 \mathrm{~min}$. The PCR products were examined by electrophoresis using a $4 \%$ agarose gel.

\section{Results}

Gene expression profiling of mRNAs and NATs in IM-9 cells irradiated with $X$-ray. A large number of studies have been performed to assess the difference in the expression of genes to obtain a clue for understanding DNA repair mechanisms and signal transduction mechanisms by IR. However, most of these studies are studies with mRNAs and microRNAs (16-18). The effects on NAT expression by IR are unknown to date. In the present study, in order to identify radiation-responsive mRNAs and NATs in IM-9 cells, we investigated mRNA and NAT expression profiles by microarray analysis using total RNA from the IM-9 cell line irradiated with X-ray doses ranging from 0 to $4 \mathrm{~Gy}$. Total RNA extraction and microarray analysis were performed as described in Materials and methods.

Eighteen, 33 and 106 mRNAs were found to be expressed differentially in IM-9 cells after 1,2 and 4 Gy irradiation, respectively, with a magnitude of $>2$-fold change as compared to 0 Gy. On the other hand, 10, 22 and 43 NATs were found to be expressed differentially in IM- 9 cells after 1, 2 and 4 Gy irradiation, respectively, with a magnitude of $>2$-fold change as compared to $0 \mathrm{~Gy}$.

Of the 18 mRNAs expressed after 1 Gy irradiation, 12 were up-regulated and 6 were down-regulated. Of the 33 mRNAs expressed after 2 Gy irradiation, 26 were up-regulated and 7 were down-regulated. Of the 106 mRNAs expressed after 4 Gy irradiation, 97 were up-regulated and 9 were down-regulated (data not shown). Some of the mRNAs described above have already been reported to be up-/down-regulated by $\operatorname{IR}(16,19)$, indicating that the results of the microarray analysis in the present study were essentially consistent with those reported in earlier studies.

Concerning the NATs, 3 of the 10 NATs expressed after 1 Gy irradiation were up-regulated and 7 were down-regulated. Of the 22 NATs expressed after 2 Gy irradiation, 7 were up-regulated and 15 were down-regulated. Of the 43 NATs expressed after 4 Gy irradiation, 32 were up-regulated and 11 were down-regulated (Tables II and III). We identified the up-regulated and down-regulated NATs caused by radiation exposure for the first time.

When it was examined whether common genes were present in the mRNAs and NATs described above, 6 genes, MDM2, CDKN1A, LSP1, PSAP, TM7SF3 and MS4A1, exhibited up-regulated mRNAs and cis-NATs with irradiation.

Strand-specific RT-qPCR analysis of MDM2 and CDKN1A $m R N A s /$ cis-NATs after X-ray irradiation. In order to confirm the expression levels detected by microarray analysis, strandspecific RT-qPCR of mRNAs and cis-NATs was carried out as described in Materials and methods. The two genes, $M D M 2$ and $C D K N 1 A$, which are shown to be involved in p53-ubiquitination and cell cycle arrest, were selected for the strand-specific RT-qPCR analysis. Since cis-NATs were found to have various initiation sites and termination sites along the genes $(15,20)$, we designed primer pairs inside the microarray probe region for strand-specific RT-qPCR (Table I).

As shown in Fig. 1, when strand-specific RT-qPCR was performed using cDNAs from mRNAs, the expression levels of MDM2 and CDKN1A mRNAs were significantly up-regulated with $\mathrm{X}$-ray dose. The up-regulation was consistent with the results of the microarray (data not shown).

Likewise, when strand-specific RT-qPCR was performed using cDNAs from cis-NATs, the expression levels of MDM2 and $C D K N 1 A$ cis-NATs were significantly up-regulated with X-ray dose (Fig. 1). The up-regulation was consistent with the results of the microarray (Table II). In addition, the microarray results and strand-specific RT-qPCR results were reported to show a good agreement in the analysis of colorectal cancer using the microarray as in the present study (13). Although, the comparisons of the present microarray system and the strand-specific RT-qPCR performed to date 
Table II. List of up-regulated NATs by IR in IM-9 cells.

\begin{tabular}{|c|c|c|c|}
\hline \multirow{2}{*}{$\begin{array}{l}\text { Radiation } \\
\text { dose }\end{array}$} & \multicolumn{3}{|c|}{ NAT profiles } \\
\hline & Accession no. & Gene name & $\begin{array}{c}\text { Fold } \\
\text { change }\end{array}$ \\
\hline \multirow[t]{3}{*}{$1 \mathrm{~Gy}$} & NM002392.2 & Mdm2, transformed $3 \mathrm{~T} 3$ cell double minute 2, p53 binding protein (mouse) (MDM2) & 2.25 \\
\hline & NM002907.2 & RecQ protein-like (DNA helicase Q1-like) (RECQL), transcript variant 1 & 2.10 \\
\hline & NM194295.1 & Chromosome 15 open reading frame $51(\mathrm{C} 15$ orf 51$)$ & 2.09 \\
\hline \multirow[t]{7}{*}{2 Gy } & NM002392.2 & Mdm2, transformed 3T3 cell double minute 2, p53 binding protein (mouse) (MDM2) & 2.94 \\
\hline & NM145689.1 & Amyloid $\beta$ (A4) precursor protein-binding, family B, member 1 (Fe65) (APBB1) & 2.52 \\
\hline & NM000389.2 & Cyclin-dependent kinase inhibitor 1A (p21, Cip1) (CDKN1A), transcript variant 1 & 2.38 \\
\hline & NM203454.1 & Apolipoprotein B mRNA editing enzyme, catalytic polypeptide-like 4 (APOBEC4) & 2.33 \\
\hline & NM152866.2 & Membrane-spanning 4-domains, subfamily A, member 1 (MS4A1), transcript variant 1 & 2.04 \\
\hline & NM194295.1 & Chromosome 15 open reading frame 51 (C15orf51) & 2.02 \\
\hline & NM002778.1 & Prosaposin (variant Gaucher disease and variant metachromatic leukodystrophy) (PSAP) & 2.02 \\
\hline \multirow[t]{32}{*}{$4 \mathrm{~Gy}$} & NM002392.2 & Mdm2, transformed 3T3 cell double minute 2, p53 binding protein (mouse) (MDM2) & 4.37 \\
\hline & XM940498.2 & PREDICTED: KIAA1430 (KIAA1430) & 3.94 \\
\hline & NM000389.2 & Cyclin-dependent kinase inhibitor 1A (p21, Cip1) (CDKN1A), transcript variant 1 & 3.71 \\
\hline & NM203454.1 & Apolipoprotein B mRNA editing enzyme, catalytic polypeptide-like 4 (APOBEC4) & 3.06 \\
\hline & NM002778.1 & Prosaposin (variant Gaucher disease and variant metachromatic leukodystrophy) (PSAP) & 2.92 \\
\hline & NM001031734.1 & Similar to RIKEN cDNA B230118G17 gene (MGC19604), transcript variant 1 & 2.84 \\
\hline & NM194295.1 & Chromosome 15 open reading frame 51 (C15orf51) & 2.72 \\
\hline & NM001039.2 & Sodium channel, nonvoltage-gated $1, \gamma(\mathrm{SCNN} 1 \mathrm{G})$ & 2.70 \\
\hline & NM015920.3 & Ribosomal protein S27-like (RPS27L) & 2.67 \\
\hline & NM078467.1 & Cyclin-dependent kinase inhibitor 1A (p21, Cip1) (CDKN1A), transcript variant 2 & 2.65 \\
\hline & NM152866.2 & Membrane-spanning 4-domains, subfamily A, member 1 (MS4A1), transcript variant 1 & 2.62 \\
\hline & NM002528.4 & Nth endonuclease III-like 1 (E. coli) (NTHL1) & 2.60 \\
\hline & NM145689.1 & Amyloid $\beta$ (A4) precursor protein-binding, family B, member 1 (Fe65) (APBB1) & 2.52 \\
\hline & NM001259.5 & Cyclin-dependent kinase 6 (CDK6) & 2.52 \\
\hline & NR003106.1 & Prader-Willi syndrome chromosome region 1 (PWCR1) on chromosome 15 & 2.49 \\
\hline & NM015464.1 & Sclerostin domain containing 1 (SOSTDC1) & 2.44 \\
\hline & NM178518.2 & Transmembrane protein 102 (TMEM102) & 2.39 \\
\hline & XR018656.1 & PREDICTED: similar to olfactory receptor 10R2 (LOC649307) & 2.26 \\
\hline & NM001023564.1 & Cathepsin L-like protein (HCTSL-s) & 2.22 \\
\hline & NM016551.1 & Transmembrane 7 superfamily member 3 (TM7SF3) & 2.22 \\
\hline & NM001610.1 & Acid phosphatase 2, lysosomal (ACP2) & 2.21 \\
\hline & NM001013255.1 & Lymphocyte-specific protein 1 (LSP1), transcript variant 4 & 2.18 \\
\hline & XM001132454.1 & PREDICTED: solute carrier organic anion transporter family, member 3A1 (SLCO3A1) & 2.18 \\
\hline & XM001129570.1 & PREDICTED: RPE-spondin (RPESP) & 2.18 \\
\hline & NM052880.3 & HGFL gene (MGC17330) & 2.16 \\
\hline & NM000825.2 & Gonadotropin-releasing hormone 1 (luteinizing-releasing hormone) (GNRH1) & 2.14 \\
\hline & NM001175.4 & Rho GDP dissociation inhibitor (GDI) $\beta$ (ARHGDIB) & 2.10 \\
\hline & NM017617.2 & Notch homolog 1, translocation-associated (Drosophila) (NOTCH1) & 2.08 \\
\hline & NM002218.3 & Inter- $\alpha$ (globulin) inhibitor H4 (plasma Kallikrein-sensitive glycoprotein) (ITIH4) & 2.08 \\
\hline & NM001004059.1 & Olfactory receptor, family 4 , subfamily $\mathrm{S}$, member 2 (OR4S2) & 2.07 \\
\hline & NM014172.2 & Phosphohistidine phosphatase 1 (PHPT1) & 2.06 \\
\hline & NR002765.1 & DDEF1 intronic transcript 1 (DDEF1IT1) on chromosome 8 & 2.03 \\
\hline
\end{tabular}

were still limited, it is strongly indicated that the results of the microarray represented the real expression profiles in IM-9 cells.
Analysis of the intracellular localization of MDM2 and CDKNIA cis-NATs. In order to obtain additional features of cis-NATs to understand their function, we selected MDM2 and 
Table III. List of down-regulated NATs by IR in IM-9 cells.

\begin{tabular}{|c|c|c|c|}
\hline \multirow{2}{*}{$\begin{array}{l}\text { Radiation } \\
\text { dose }\end{array}$} & \multicolumn{3}{|c|}{ NAT profiles } \\
\hline & Accession no. & Gene name & $\begin{array}{l}\text { Fold } \\
\text { change }\end{array}$ \\
\hline \multirow[t]{7}{*}{$1 \mathrm{~Gy}$} & XM001130893.1 & PREDICTED: hypothetical protein LOC729643 (LOC729643) & 6.76 \\
\hline & XM001129283.1 & PREDICTED: hypothetical protein LOC729087 (LOC729087) & 2.84 \\
\hline & NM153020.1 & RNA binding motif protein 24 (RBM24) & 2.54 \\
\hline & NM017917.2 & Chromosome 14 open reading frame 10 (C14orf10) & 2.37 \\
\hline & NM182605.1 & Hypothetical protein FLJ40448 (FLJ40448) & 2.13 \\
\hline & NM001005218.1 & Olfactory receptor, family 5 , subfamily $\mathrm{B}$, member 21 (OR5B21) & 2.05 \\
\hline & NM005247.2 & Fibroblast growth factor 3 (FGF3) & 2.02 \\
\hline \multirow[t]{15}{*}{2 Gy } & XM001130893.1 & PREDICTED: hypothetical protein LOC729643 (LOC729643) & 5.48 \\
\hline & NM153020.1 & RNA binding motif protein 24 (RBM24) & 2.89 \\
\hline & XM001129283.1 & PREDICTED: hypothetical protein LOC729087 (LOC729087) & 2.88 \\
\hline & NM005247.2 & Fibroblast growth factor 3 (FGF3) & 2.58 \\
\hline & NM015015.1 & Jumonji domain containing 2B (JMJD2B) & 2.50 \\
\hline & NM182605.1 & Hypothetical protein FLJ40448 (FLJ40448) & 2.39 \\
\hline & NM003532.2 & Histone 1, H3e (HIST1H3E) & 2.38 \\
\hline & NM025109.4 & Myosin head domain containing 1 (MYOHD1), transcript variant 1 & 2.26 \\
\hline & NM001005218.1 & Olfactory receptor, family 5 , subfamily $\mathrm{B}$, member 21 (OR5B21) & 2.25 \\
\hline & NM003120.1 & Spleen focus forming virus (SFFV) proviral integration oncogene spi1 (SPI1) & 2.19 \\
\hline & NM206965.1 & Formiminotransferase cyclodeaminase (FTCD), transcript variant A & 2.15 \\
\hline & NM001039661.1 & $\begin{array}{l}\text { Toll-interleukin } 1 \text { receptor (TIR) domain containing adaptor protein (TIRAP), } \\
\text { transcript variant } 3\end{array}$ & 2.11 \\
\hline & NM001004490.1 & Olfactory receptor, family 2 , subfamily AG, member 2 (OR2AG2) & 2.07 \\
\hline & NM000819.3 & $\begin{array}{l}\text { Phosphoribosylglycinamide formyltransferase, phosphoribosylglycinamide synthetase, } \\
\text { phosphoribosylaminoimidazole synthetase (GART), transcript variant } 1\end{array}$ & 2.05 \\
\hline & NM024819.3 & Dephospho-CoA kinase domain containing (DCAKD) & 2.03 \\
\hline \multirow[t]{11}{*}{4 Gy } & XM001130893.1 & PREDICTED: hypothetical protein LOC729643 (LOC729643) & 4.70 \\
\hline & XM001129283.1 & PREDICTED: hypothetical protein LOC729087 (LOC729087) & 2.83 \\
\hline & NM182605.1 & Hypothetical protein FLJ40448 (FLJ40448) & 2.25 \\
\hline & NM003545.3 & Histone $1, \mathrm{H} 4 \mathrm{e}$ (HIST1H4E) & 2.24 \\
\hline & NM175055.2 & Histone $3, \mathrm{H} 2 \mathrm{bb}$ (HIST3H2BB) & 2.20 \\
\hline & NM003543.3 & Histone 1, H4h (HIST1H4H) & 2.19 \\
\hline & NM080596.1 & Histone 1, H2ah (HIST1H2AH) & 2.15 \\
\hline & NM002467.3 & v-myc myelocytomatosis viral oncogene homolog (avian) (MYC) & 2.12 \\
\hline & NM003513.2 & Histone 1, H2ab (HIST1H2AB) & 2.12 \\
\hline & NM175065.2 & Histone 2, H2ab (HIST2H2AB) & 2.02 \\
\hline & NM017917.2 & Chromosome 14 open reading frame $10(\mathrm{C} 14$ orf 10$)$ & 2.02 \\
\hline
\end{tabular}

CDKN1A cis-NATs as an example, and examined the intracellular localization of these $c$ is-NATs in IM-9 cells.

Total RNA, cytoplasmic RNA and nuclear RNA fractions were prepared from IM-9 cells. As shown in Fig. 2A, 18 and $28 \mathrm{~S}$ ribosomal RNAs were detected in the cytoplasmic fraction, but not in the nuclear fraction, indicating that preparation of RNA from the cytoplasm and nucleus functioned well. The RNA fractions thus obtained were examined to ascertain whether they contain the cis-NATs. As shown in Fig. 2B, PCR using cDNAs from mRNAs demonstrated that the MDM2 and CDKN1A mRNAs were detected in the cytoplasmic fraction. Concerning cis-NATs, the MDM2 cis-NATs were detected in the cytoplasmic fraction, and the CDKN1A cis-NATs was detected in both the nuclear and cytoplasmic fraction. These results suggest that $c$ is-NATs of $M D M 2$ and $C D K N 1 A$ have a different function in IM-9 cells.

\section{Discussion}

Ionizing radiation is frequently used for the diagnosis and treatment of various types of cancer in the medical field, but accidental exposure may result. In the present study, we examined the effect of ionizing radiation on cells through the analysis of expression profiles of mRNAs/cis-NATs in IM-9 cells irradiated by X-ray, using a custom microarray. Eighteen, 33 and 

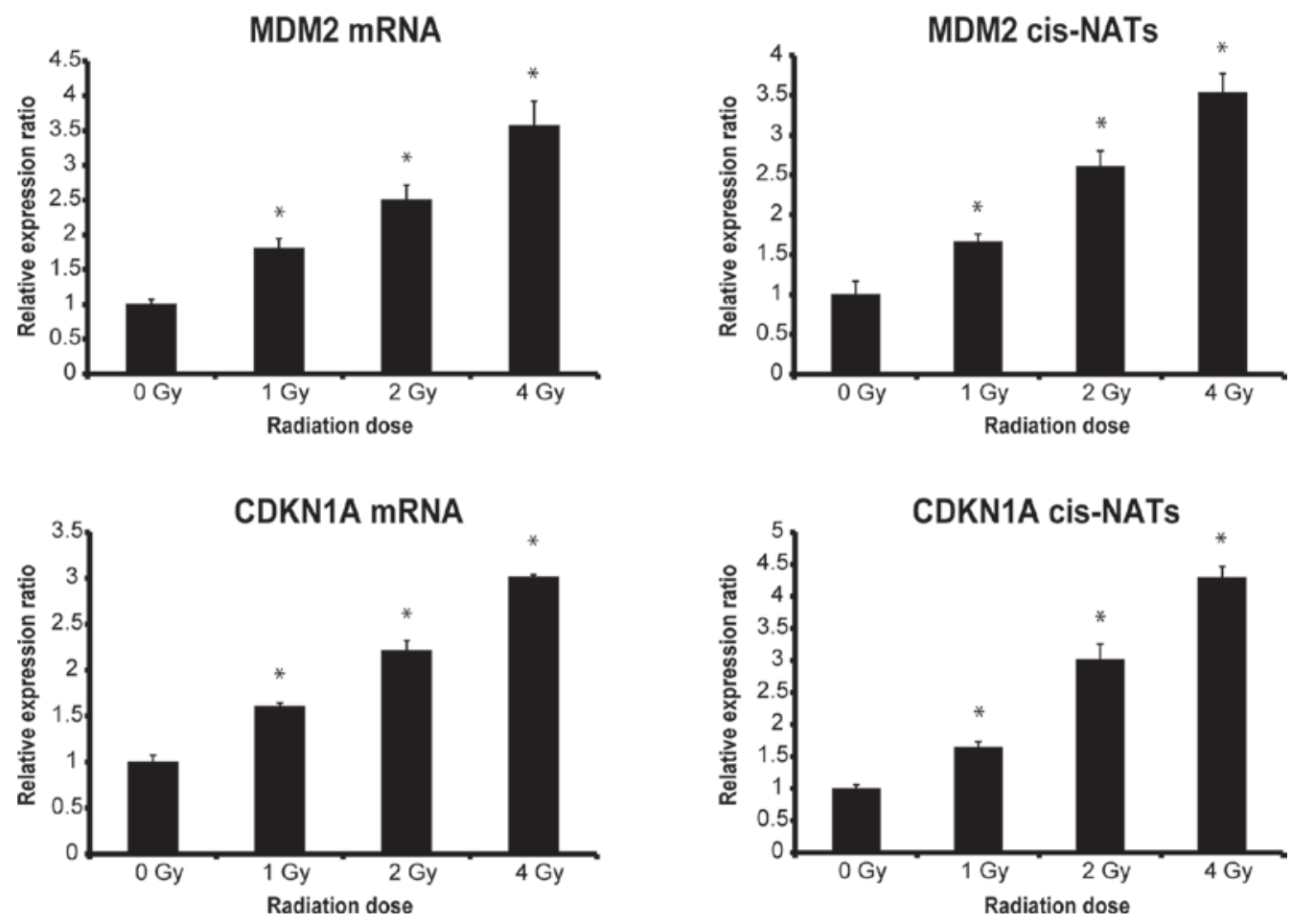

Figure 1. Relative expression of $M D M 2$ and $C D K N 1 A$ mRNAs/cis-NATs after X-ray irradiation in IM-9 cells. Total RNAs were isolated from irradiated and non-irradiated IM-9 cells as described in Materials and methods. The mRNA and cis-NAT expression levels were determined using strand-specific RT-qPCR analysis. The values for mRNAs and cis-NATs were normalized based on values of the ACTB mRNA. "Statistical significance, compared to 0 Gy ( $<<0.05$ ).
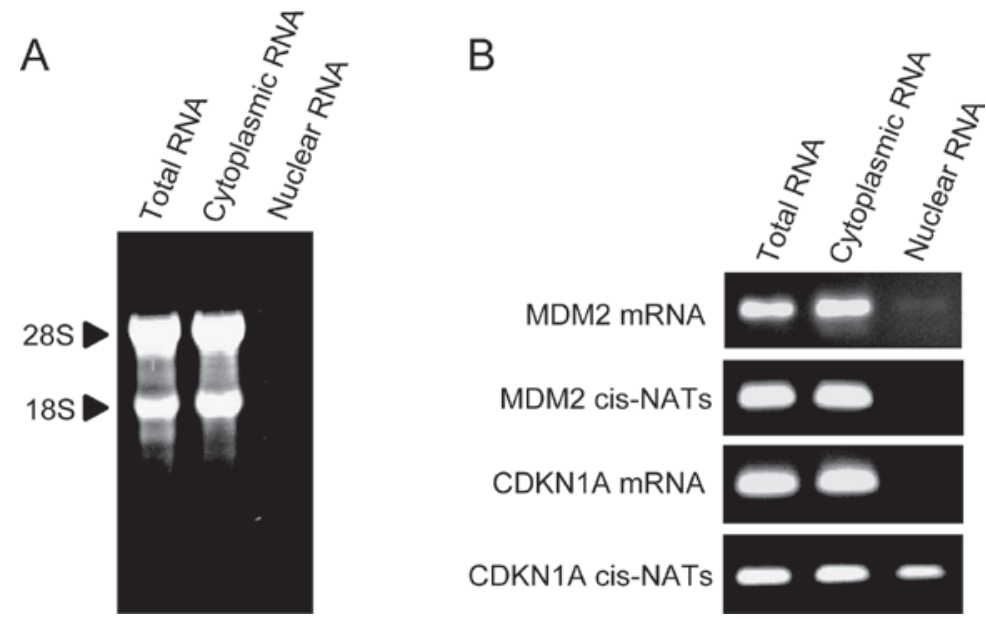

Figure 2. Intracellular localization of $M D M 2$ and $C D K N 1 A$ mRNAs/cis-NATs in IM-9 cells. (A) Isolation of the cytoplasmic and nuclear fraction of RNA. Total RNA, cytoplasmic RNA and nuclear RNA were isolated using PARIS kit. (B) Strand-specific RT-PCR for intracellular localization of mRNAs and cis-NATs. Strand-specific cDNAs derived from mRNAs or cis-NATs were synthesized using reverse or forward primer (Table I), and PCRs were performed as described in Materials and methods. The amplified fragments were electrophoresed on $4 \%$ agarose gel.

106 mRNAs were demonstrated to be differentially expressed in the IM-9 cells after 1, 2 and 4 Gy irradiation, respectively, as compared to $0 \mathrm{~Gy}$ by microarray analysis ( $\mathrm{FC}>2.0)$. On the other hand, 10, 22 and 43 cis-NATs were demonstrated to be differentially expressed in the IM-9 cells after 1, 2 and 4 Gy irradiation, respectively, as compared to 0 Gy by microarray analysis (FC > 2.0). MDM2 and CDKN1A, which are involved in the regulation of the cell cycle through $\mathrm{p} 53$, were selected as probes for verification of the results of the microarray analysis using strand-specific RT-qPCR. The PCR provided supportive evidence for the results of the microarray. In addition, the
mRNA expression levels elevated by X-ray exposure in the present study were found to include those such as MDM2 and $C D K N 1 A$ as reported earlier $(16,19)$. Collectively, these findings strongly indicate that the expression profiles obtained in the present study represent the expression of transcripts in the cells.

Morris et al demonstrated that the cis-NAT of CDKN1A induces trimethylation of Lys27 of Histone 3 (H3K27me3) to reduce the expression levels of $C D K N 1 A$ mRNA (21). This function of $C D K N 1 A$ cis-NAT is considered to be restricted to the nucleus. However, since the $c i s$-NAT of $C D K N 1 A$ was 
found not only in the nucleus, but also in the cytoplasm, the cis-NAT should have additional functions in the cytoplasm. As for the $c i s$-NAT of MDM2, the cis-NAT was found only in the cytoplasm, indicating that its function is different from that of the CDKN1A cis-NAT.

In conclusion, the radiation-responsive cis-NATs in conjunction with mRNAs were identified for the first time in the present study. It is possible that these cis-NATs regulate the gene expression in a post-transcriptional fashion. Currently, the function of cis-NAT in ionizing radiation has been unknown. However, the expression profiles of mRNAs/cis-NATs may be used as markers for the amount of ionizing radiation which cells receive. In order to clarify the function of cis-NATs in $\mathrm{X}$-ray exposure, the products of the respective genes should first be examined in terms of their amounts in future studies.

\section{Acknowledgements}

The authors are grateful to Dr Yoshiaki Nagamura and Ms. Ritsuko Motoyama, National Institute of Agrobiological Sciences, Tsukuba, for the excellent support in the mirroarray analysis. The present study was supported by a grant from the Hirosaki University Graduate School of Health Sciences Radiation Emergency Medicine Human Resource Cultivation Project.

\section{References}

1. Ziegler M and Oei SL: A cellular survival switch: poly(ADPribosyl)ation stimulates DNA repair and silences transcription. Bioessays 23: 543-548, 2001.

2. Jackson SP: DNA-dependent protein kinase. Int J Biochem Cell Biol 29: 935-938, 1997.

3. Bourguignon MH, Gisone PA, Perez MR, et al: Genetic and epigenetic features in radiation sensitivity Part I: cell signaling in radiation response. Eur J Nucl Med Mol Imaging 32: 229-246, 2005.

4. Levine AJ: p53, the cellular gatekeeper for growth and division. Cell 88: 323-331, 1997.

5. Samuel T, Weber HO, Rauch P, et al: The G2/M regulator 14-3-3 sigma prevents apoptosis through sequestration of Bax. J Biol Chem 276: 45201-45206, 2001.

6. Rugo RE, Secretan MB and Schiestl RH: X radiation causes a persistent induction of reactive oxygen species and a delayed reinduction of TP53 in normal human diploid fibroblasts. Radiat Res 158: 210-219, 2002.
7. Rosok O and Sioud M: Systematic identification of senseantisense transcripts in mammalian cells. Nat Biotechnol 22: 104-108, 2004.

8. Katayama S, Tomaru Y,Kasukawa T, et al: Antisense transcription in the mammalian transcriptome. Science 309: 1564-1566, 2005.

9. Werner A and Berdal A: Natural antisense transcripts: sound or silence? Physiol Genomics 23: 125-131, 2005.

10. Faghihi MA, Modarresi F, Khalil AM, et al: Expression of a noncoding RNA is elevated in Alzheimer's disease and drives rapid feed-forward regulation of beta-secretase. Nat Med 14: 723-730, 2008

11. Hatzoglou A, Deshayes F, Madry C, et al: Natural antisense RNA inhibits the expression of BCMA, a tumor necrosis factor receptor homologue. BMC Mol Biol 3: 1-7, 2002.

12. Yu W, Gius D, Onyango P, et al: Epigenetic silencing of tumour suppressor gene p15 by its antisense RNA. Nature 451: 202-206, 2008.

13. Kohno K, Chiba M, Murata S, et al: Identification of natural antisense transcripts involved in human colorectal cancer development. Int J Oncol 37: 1425-1432, 2010.

14. Grigoriadis A, Oliver GR, Tanney A, et al: Identification of differentially expressed sense and antisense transcript pairs in breast epithelial tissues. BMC Genomics 10: 324, 2009.

15. Chiba M, Kiyosawa H, Hiraiwa N, Ohkohchi $\mathrm{N}$ and Yasue $\mathrm{H}$ : Existence of Pink1 antisense RNAs in mouse and their localization. Cytogenet Genome Res 126: 259-270, 2009.

16. Kabacik S, Mackay A, Tamber N, et al: Gene expression following ionizing radiation: identification of biomarkers for dose estimation and prediction of individual response. Int $\mathrm{J}$ Radiat Biol 87: 115-129, 2011.

17. Cha HJ, Shin S, Yoo H, et al: Identification of ionizing radiationresponsive microRNAs in the IM9 human B lymphoblastic cell line. Int J Oncol 34: 1661-1668, 2009.

18. Cha HJ, Seong KM, Bae S, et al: Identification of specific microRNAs responding to low and high dose gamma-irradiation in the human lymphoblast line IM9. Oncol Rep 22: 863-868, 2009.

19. Turtoi A, Brown I, Oskamp D and Schneeweiss FH: Early gene expression in human lymphocytes after gamma-irradiation a genetic pattern with potential for biodosimetry. Int J Radiat Biol 84: 375-387, 2008.

20. Kiyosawa H, Mise N, Iwase S, Hayashizaki Y and Abe K: Disclosing hidden transcripts: mouse natural sense-antisense transcripts tend to be poly(A) negative and nuclear localized. Genome Res 15: 463-474, 2005.

21. Morris KV, Santoso S, Turner AM, Pastori C and Hawkins PG: Bidirectional transcription directs both transcriptional gene activation and suppression in human cells. PLoS Genet 4: e1000258, 2008. 